
\title{
Condições de trabalho de profissionais da enfermagem:
avaliação baseada no modelo demanda-controle*
}

\author{
Working conditions of nurses: evaluation based on the demand-control model
}

\author{
Condiciones de trabajo de profesionales de enfermería: evaluación basada en el modelo demanda- \\ control
}

\section{Tânia Solange Bosi de Souza Magnago', Marcia Tereza Luz Lisboa², Rosane Harter Griep ${ }^{3}$, Regina Célia Gollner Zeitoune ${ }^{4}$, Juliana Petri Tavares ${ }^{5}$}

\section{RESUMO}

Objetivo: Descrever a relação entre os indicadores de demandas psicológicas e de controle sobre o trabalho entre trabalhadores de enfermagem, segundo o Modelo Demanda-Controle. Métodos: Estudo seccional, com 491 trabalhadores de enfermagem de um hospital universitário público do Rio Grande do Sul, no período de março a dezembro de 2006. Utilizou-se a versão brasileira do "Job Content Questionnaire”, para avaliação das dimensões psicossociais do trabalho (demanda psicológica e controle). Resultado: Obteve-se 30\% dos trabalhadores de enfermagem classificados no grupo trabalho passivo (baixa demanda e baixo controle), 29\% no grupo trabalho ativo (alta demanda e alto controle), $21 \%$ no grupo alta exigência (alta demanda e baixo controle) e 20\% no grupo baixa exigência (baixa demanda e alto controle). A alta exigência foi maior entre técnicos e auxiliares de enfermagem. Conclusão: Medidas que visem a reduzir as demandas psicológicas provenientes do trabalho e que permitam maior flexibilidade e autonomia ao trabalhador são necessárias.

Descritores: Condições de trabalho; Saúde do trabalhador; Recursos humanos de Enfermagem/psicologia

\begin{abstract}
Objective: To describe the relationship between indicators of psychological demands and job control among nursing workers, according to the Demand-Control Model. Methods: It was a cross-sectional study, with 491 nurses of a public university hospital in Rio Grande do Sul, in the period of March to December 2006. It was used the Brazilian version of the Job Content Questionnaire, to assess the psychosocial dimensions of work (psychological demands and control). Results: It was found that $30 \%$ of nursing workers were classified in the passive work group (low demand and low control), 29\% in the active work (high demand and high control), 21\% in the high demand ( high demand and low control) and 20\% in the low demand (low demand and high control). The high demand was higher among technicians and nursing assistants. Conclusion: It is necessary to implement measures that aim to reduce the psychological demands from work and allow greater flexibility and autonomy to the worker.
\end{abstract}

Keywords: Working conditions; Occupational health; Nursing staff/psychology

\section{RESUMEN}

Objetivo: Describir la relación entre los indicadores de demandas psicológicas y de control sobre el trabajo entre trabajadores de enfermería, según el Modelo Demanda-Control. Métodos: Estudio seccional, con 491 trabajadores de enfermería de un hospital universitario público de Rio Grande do Sul, en el período de marzo a diciembre de 2006. Se utilizó la versión brasileña del Job Content Questionnaire, para evaluar las dimensiones psicosociales del trabajo (demanda psicológica y control). Resultado: Se obtuvo que 30\% de los trabajadores de enfermería fueron clasificados en el grupo trabajo pasivo (baja demanda y bajo control), $29 \%$ en el grupo trabajo activo (alta demanda y alto control), $21 \%$ en el grupo alta exigencia (alta demanda y bajo control) y $20 \%$ en el grupo baja exigencia (baja demanda y alto control). La alta exigencia fue mayor entre técnicos y auxiliares de enfermería. Conclusión: Son necesarias medidas que tengan por objetivo reducir las demandas psicológicas provenientes del trabajo y que permitan mayor flexibilidad y autonomía al trabajador.

Descriptores: Condiciones de trabajo; Salud laboral; Personal de Enfermería/psicología

* Trabalho extraído da Tese de Doutorado "Aspectos psicossociais do trabalho e distúrbio musculoesquelético em trabalhadores de enfermagem" apresentada ao Programa de Pós-graduação em Enfermagem da Escola de Enfermagem Anna Nery - UFRJ, Brasil. Bolsa PQI Capes. Estudo realizado em um hospital universitário público do Rio Grande do Sul (RS), Brasil.

${ }^{1}$ Doutora em Enfermagem. Professora Adjunto do Departamento de Enfermagem da Universidade Federal de Santa Maria - UFSM - Santa Maria (RS), Brasil.

${ }^{2}$ Doutora em Enfermagem. Professora Associada do Departamento de Enfermagem Fundamental da Escola de Enfermagem Anna Nery da Universidade Federal do Rio de Janeiro - UFRJ - Rio de Janeiro (RJ), Brasil.

${ }^{3}$ Doutora em Ciências. Pesquisadora do Laboratório de Educação em Saúde e Ambiente da Fundação Osvaldo Cruz - FIOCRUZ, Rio de Janeiro (RJ), Brasil. ${ }^{4}$ Doutora em Enfermagem. Professora Adjunto do Departamento de Enfermagem de Saúde Pública da Escola de Enfermagem Anna Nery da Universidade Federal do Rio de Janeiro - UFRJ - Rio de Janeiro (RJ), Brasil.

${ }^{5}$ Pós-graduanda (Mestrado) do Programa de Pós-Graduação em Enfermagem da Universidade Federal de Santa Maria - UFSM - Santa Maria (RS), Brasil. 


\section{INTRODUÇÃO}

A enfermagem é classificada como uma das ocupações da área da saúde com alto risco de tensão e adoecimento $^{(1-3)}$. Se o trabalho pode ser considerado como gerador de desgaste e tensão, o estresse ocupacional pode acontecer, quando há um desequilíbrio resultante da relação entre demandas no trabalho e a habilidade efetiva do trabalhador no enfrentamento das situações estressoras $^{(3)}$. O sofrimento psicológico do trabalhador pode acontecer quando ele já não consegue transformar seu trabalho, no sentido de buscar adequá-lo às suas necessidades psíquicas e fisiológicas ${ }^{(4)}$.

A lista de estressores no trabalho da enfermagem hospitalar é ampla, conferindo-lhe condições laborais inadequadas. Dentre os aspectos negativos do trabalho da enfermagem, destacam-se: a rotina das tarefas; as interrupções constantes; os conflitos interpessoais com administradores, médicos e a própria equipe de enfermagem; dificuldade em responder às demandas emocionais dos pacientes e seus familiares; contato com a dor e a morte; deficit no quadro de pessoal; tempo livre insuficiente para atividades extralaborais; ritmo de trabalho intenso e não participação na tomada de decisão sobre mudanças institucionais ${ }^{(5)}$.

Essa lista aponta para as cargas de trabalho da enfermagem $^{(4)}$ e evidencia um grupo de estressores psicossociais $^{(6)}$, frequentemente, associados à ocorrência de doenças ocupacionais. Dentre os modelos que caracterizam o estresse psicossocial no trabalho, destacase o Modelo Demanda-Controle (MDC), proposto no final da década de 1970. Segundo esse Modelo, altas demandas psicológicas e baixo controle do trabalhador sobre o trabalho poderiam se constituir em indicadores de sobrecarga física e emocional no cotidiano laboral ${ }^{(6)}$.

O MDC distingue quatro tipos básicos de experiência no trabalho, gerados pela interação dos níveis de demandas psicológicas e de controle: baixa exigência (baixa demanda e alto controle-categoria de referência), trabalho ativo (alta demanda e baixo controle), trabalho passivo (baixa demanda e baixo controle) e alta exigência (alta demanda e baixo controle - categoria de maior exposição a adoecimentos) ${ }^{(6)}$. Este Modelo vem sendo aplicado em vários países, sobretudo na Europa, com a finalidade de analisar as dimensões psicossociais no contexto laboral ${ }^{(5-7)}$.

Tendo por base os quatro grupos de trabalho propostos no MDC, estabeleceram-se as seguintes questões norteadoras deste estudo: qual o nível de demanda psicológica e de controle sobre o trabalho dos trabalhadores de enfermagem de um hospital público universitário do Rio Grande do Sul? Qual a frequência desses trabalhadores em cada grupo do MDC?

Assinala-se como objeto de estudo as demandas psicológicas do trabalho e o controle autorreferidos pelos trabalhadores de enfermagem. Com base no conhecimento do nível e da relação entre essas variáveis, podem ser sugeridas medidas de flexibilização na organização do trabalho, de forma a reduzir a sobrecarga psicossomática e, consequentemente, evitar adoecimentos resultantes das agressões sofridas no contexto organizacional.

Este estudo teve por objetivo descrever a relação entre os indicadores de demandas psicológicas e de controle sobre o trabalho entre trabalhadores de enfermagem, segundo o MDC.

\section{MÉTODOS}

Trata-se de um estudo epidemiológico seccional, realizado em um hospital universitário público da Região Centro-Oeste do Rio Grande do Sul, centro de referência em atendimento à urgência e emergência, tratamento intensivo e transplante de medula óssea. Do total de trabalhadores de enfermagem (528), 491 participaram do estudo $(93 \%)$. As perdas $(7 \%)$ resultaram de recusas e aposentadorias. Utilizou-se um questionário com perguntas fechadas preestabelecidas, contendo as seguintes variáveis: Sociodemográficas, idade, sexo, escolaridade, situação conjugal, renda familiar per capita em salários mínimos, Índice de Massa Corpórea-IMC e Tabagismo; Laborais: função (enfermeiro; técnico ou auxiliar de enfermagem), setor, tempo na função e no setor, carga horária semanal, turno; Dimensãoes psicossociais do trabalho (versão brasileira do Job Content Questionnaire - JCQ ${ }^{(5,7)}$.

A coleta dos dados foi feita por meio de entrevista durante o turno de trabalho, no período de março a dezembro de 2006, por entrevistadores previamente capacitados. Para a inserção dos dados, foi utilizado o programa Epi-infoß, versão 6.04, com dupla digitação independente. Após a verificação de erros e inconsistências, a análise dos dados foi realizada no programa SPSS $® 18.0$ for windows. As escalas para demanda psicológica e controle foram calculadas, usando as recomendações do manual do JCQ (www.jcqcenter.org). Os quadrantes do MDC foram construídos, baseados na mediana da distribuição e dicotomizados em níveis de baixo/alto controle e baixa/ alta demanda, formando os grupos: baixa exigência (grupo de referência), trabalho passivo, trabalho ativo e alta exigência (grupo de maior exposição). Considerou-se associação significativa entre as variáveis estudadas quando $\mathrm{p}<0,05$. Os coeficientes de Alpha de Cronbach para demanda psicológica e controle foram, respectivamente, 0,70 e 0,59. O estudo foi aprovado pelo Comitê de Ética em Pesquisa da instituição proponente (CAAE 0008.0.243.000-07). Os participantes assinaram o Termo de Consentimento Livre e Esclarecido após terem sido esclarecidos sobre os aspectos da pesquisa. 


\section{RESULTADOS}

Caracterização sociodemográfica dos participantes: os trabalhadores de enfermagem eram predominantemente do sexo feminino $(88,4 \%)$; a média da idade foi 41 anos ( $\pm 8,7$ anos); idade mínima de 22 anos e máxima de 67 anos; a maioria era casada ou vivia com companheiros $(65,8 \%)$ e $58,9 \%$ tinham ensino médio. É importante ressaltar que $13 \%$ dos técnicos de enfermagem possuíam graduação em enfermagem. Com relação à renda familiar per capita, $41,3 \%$ dos trabalhadores foram classificados em menos de dois salários mínimos. Identificaram-se $34 \%$ dos trabalhadores com sobrepeso e $14,1 \%$ com obesidade; $10,8 \%$ eram fumantes.

Caracterização laboral dos participantes: do total de 491 trabalhadores, 29,7\% eram enfermeiros, 32,8\% técnicos de enfermagem e $37,5 \%$ auxiliares de enfermagem. Esses profissionais trabalhavam na função atual em média há 14,4 anos ( $\pm 8,3$ anos). Os setores que mais concentraram trabalhadores foram as Unidades de Clínicas (Internação clínica, Internação cirúrgica, Hematooncologia e Psiquiatria) - 32,6\%, Terapia Intensiva (adulto, pediátrica e neonatal) - 19,1\%, Materno infantil - 14,1\%, seguidas pelas Unidades Cirúrgica e de Urgência e Emergência com respectivamente $11,8 \%$ e $11,4 \%$ dos trabalhadores. Os enfermeiros, técnicos e auxiliares trabalhavam no setor atual em média há 8 anos. Ainda, do total de trabalhadores, 59,3\% trabalhavam em turno diurno; $53 \%$ desenvolviam carga horária de 36 horas semanais e $26,3 \%$ dos trabalhadores de enfermagem referiram outro vínculo de trabalho.

Caracterização das dimensões psicossociais do trabalho: as dimensões controle sobre o trabalho e demanda psicológica, segundo categoria profissional foram, em um primeiro momento, avaliadas individualmente. Os resultados são apresentados nas Tabelas 1 a 4. Num segundo momento, foram agrupadas de acordo com os quadrantes do MDC (Tabela 5).

Ao ser analisar os dados da Tabela 1, referentes à dimensão psicossocial controle sobre o trabalho, as diferenças dos percentuais foram significativas para a maioria das questões abordadas. Os enfermeiros obtiveram percentuais mais elevados nos seguintes itens: o trabalho requerer que aprendam coisas novas, que sejam criativos, exige um alto nível de habilidade, poder fazer muitas coisas diferentes, tomar muitas decisões por própria conta e dar opiniões, e elas serem aceitas. $\mathrm{O}$ mesmo não aconteceu com a categoria de técnicos e auxiliares de enfermagem. Além dos menores percentuais para as questões que conferem alto controle, obtiveram percentuais elevados para trabalho que envolve muita repetitividade e pouca liberdade para decidir como fazê-lo.

A Tabela 2 evidencia diferença significativa entre os grupos avaliados no que tange ao grau de controle no trabalho $(\mathrm{p}=0,000)$.

Ao analisar os dados da Tabela 3, em relação aos indicadores de demandas psicológicas, pode-se observar que os técnicos e auxiliares apresentaram percentuais mais elevados do que os enfermeiros nas questões em que o trabalho envolve volume excessivo de tarefas e menor exposição a conflitos. No caso das duas primeiras questões - trabalho rápido e duro, não houve diferenças significativas entre as categorias profissionais. $\mathrm{Na}$ Tabela 4 , evidencia-se maior percentual de enfermeiros classificados com maior demanda psicológica no trabalho do que os técnicos/auxiliares de enfermagem $(p<0,001)$.

Tabela 1 - Indicadores de controle exercido sobre o próprio trabalho referido pelos trabalhadores de enfermagem de um hospital universitário. RS, 2006.

\begin{tabular}{|c|c|c|c|c|c|}
\hline \multirow{3}{*}{ Indicadores de controle } & \multicolumn{4}{|c|}{ Função } & \multirow{3}{*}{$\begin{array}{l}\text { Valor } \\
\text { de } p\end{array}$} \\
\hline & \multicolumn{2}{|c|}{ Enfermeiro } & \multicolumn{2}{|c|}{ Técnico/auxiliar } & \\
\hline & $\mathbf{n}$ & $\%$ & n & $\%$ & \\
\hline Meu trabalho requer que eu aprenda coisas novas & & & & & 0,000 \\
\hline Concordo & 146 & 100,0 & 335 & 97,1 & \\
\hline Meu trabalho envolve muita repetitividade & & & & & 0,109 \\
\hline Concordo & 124 & 84,9 & 313 & 90,7 & \\
\hline Meu trabalho requer que eu seja muito criativo & & & & & 0,000 \\
\hline Concordo & 131 & 89,7 & 267 & 77,4 & \\
\hline Meu trabalho permite que eu tome muitas decisões por minha própria conta & & & & & 0,000 \\
\hline Concordo & 122 & 83,6 & 223 & 64,6 & \\
\hline Meu trabalho exige um alto nível de habilidade & & & & & 0,000 \\
\hline Concordo & 140 & 95,9 & 309 & 89,6 & \\
\hline Em meu trabalho tenho pouca liberdade para decidir como eu devo fazê-lo & & & & & 0,000 \\
\hline Concordo & 127 & 87,0 & 326 & 94,5 & \\
\hline Em meu trabalho posso fazer muitas coisas diferentes & & & & & 0,000 \\
\hline Concordo & 105 & 71,9 & 183 & 53,0 & \\
\hline O que tenho a dizer sobre o que acontece no meu trabalho é considerado & & & & & 0,000 \\
\hline Concordo & 128 & 87,7 & 282 & 81,7 & \\
\hline Tenho oportunidade de desenvolver minhas habilidades especiais & & & & & 0,006 \\
\hline Concordo & 121 & 82,9 & 245 & 71,0 & \\
\hline
\end{tabular}


Tabela 2 - Distribuição das categorias profissionais de enfermagem segundo o grau de controle sobre o processo de trabalho em um hospital universitário. RS, 2006.

\begin{tabular}{lrrrrr}
\hline \multirow{3}{*}{ Categorias } & \multicolumn{3}{c}{ Controle sobre o trabalho } & \multirow{2}{*}{ Valor de $\boldsymbol{p}$} \\
\cline { 2 - 5 } & \multicolumn{3}{c}{ Baixo } & \multicolumn{3}{c}{ Alto } & \\
\cline { 2 - 5 } & $\mathbf{n . ~}^{\mathbf{o}}$ & $\mathbf{\%}$ & $\mathbf{n .}^{\mathbf{0}}$ & $\mathbf{\%}$ & \\
\hline Enfermeiro & 36 & 24,7 & 110 & 75,3 & 0,000 \\
Técnico/auxiliar de & 215 & 62,3 & 130 & 37,7 & \\
enfermagem & & & & & \\
\hline
\end{tabular}

Tabela 3 - Indicadores de demandas psicológicas do trabalho referida pelos trabalhadores de enfermagem em um hospital universitário. RS, 2006.

\begin{tabular}{|c|c|c|c|c|c|}
\hline \multirow{3}{*}{$\begin{array}{l}\text { Questões indicadoras de } \\
\text { demanda Psicológica }\end{array}$} & \multicolumn{4}{|c|}{ Função } & \multirow{3}{*}{$\begin{array}{l}\text { Valor } \\
\text { de } p\end{array}$} \\
\hline & \multicolumn{2}{|c|}{ Enfermeiro } & \multicolumn{2}{|c|}{$\begin{array}{l}\text { Técnico e } \\
\text { auxiliar }\end{array}$} & \\
\hline & $\mathrm{n}$ & $\%$ & $\mathrm{n}$ & $\%$ & \\
\hline $\begin{array}{l}\text { Meu trabalho requer que } \\
\text { eu trabalhe muito } \\
\text { rapidamente }\end{array}$ & & & & & 0,381 \\
\hline Concordo & 95 & 65,1 & 210 & 60,9 & \\
\hline $\begin{array}{l}\text { Meu trabalho requer que } \\
\text { eu trabalhe muito duro }\end{array}$ & & & & & 0,930 \\
\hline Concordo & 73 & 50,0 & 171 & 49,6 & \\
\hline $\begin{array}{l}\text { Eu não sou solicitado para } \\
\text { realizar um volume } \\
\text { excessivo de trabalho }\end{array}$ & & & & & 0,020 \\
\hline Concordo & 47 & 32,2 & 150 & 43,5 & \\
\hline $\begin{array}{l}\text { O tempo para a realização } \\
\text { das minhas tarefas é } \\
\text { suficiente }\end{array}$ & & & & & 0,002 \\
\hline Concordo & 97 & 66,4 & 275 & 79,7 & \\
\hline $\begin{array}{l}\text { Estou livre de demandas } \\
\text { conflitantes feitas por } \\
\text { outros }\end{array}$ & & & & & 0,000 \\
\hline Concordo & 38 & 26,0 & 185 & 53,6 & \\
\hline
\end{tabular}

Tabela 4 - Categorias profissionais de enfermagem segundo grau de demanda psicológica no trabalho em um hospital universitário. RS, 2006.

\begin{tabular}{lrrrrr}
\hline \multirow{2}{*}{ Categorias } & \multicolumn{4}{c}{ Demanda psicológica } & \multirow{2}{*}{ Valor } \\
& \multicolumn{2}{c}{ Baixa } & \multicolumn{2}{c}{ Alta } & de $\boldsymbol{p}$ \\
\cline { 2 - 5 } & 56 & 38,4 & 90 & 61,6 & $<0,001$ \\
\hline Enfermeiro & 191 & 55,4 & 154 & 44,6 & \\
$\begin{array}{l}\text { Técnico/auxiliares de } \\
\text { enfermagem }\end{array}$ & & & & \\
\hline
\end{tabular}

Tabela 5 - Freqüência de trabalhadores de enfermagem de um hospital universitário, segundo quadrantes do modelo demanda-controle. RS, 2006.

\begin{tabular}{|c|c|c|}
\hline Quadrantes Modelo D-C & $\mathbf{n}$ & $\%$ \\
\hline Baixa exigência $(\mathbf{D} \wedge \mathrm{C})$ & 100 & 20,4 \\
\hline Trabalho passivo (WDC) & 147 & 29,9 \\
\hline Trabalho ativo (NDAC) & 140 & 28,5 \\
\hline Alta exigência (NDNC) & 104 & 21,2 \\
\hline
\end{tabular}

A análise das interações entre os níveis de demandas psicológicas e de controle sobre o trabalho revelou que os trabalhadores de enfermagem encontravam-se predominantemente na categoria de trabalho passivo $(29,9 \%)$, seguido pela categoria trabalho ativo $(28,5 \%)$ (Tabela 5).

\section{DISCUSSÃO}

Neste estudo, os enfermeiros expressaram maior grau de controle sobre o próprio trabalho do que os técnicos e auxiliares de enfermagem, possivelmente pela divisão hierarquizada do trabalho da enfermagem. Por outro lado, os enfermeiros referiram mais características negativas do trabalho, no que tange à maior demanda psicológica.

Dois fatos relacionados à dimensão controle chamam à atenção neste estudo. O primeiro, apesar de historicamente a divisão do trabalho favorecer o enfermeiro, pois a ele compete supervisionar, ensinar e coordenar a equipe de enfermagem, cerca de $25 \%$ deles foram classificados no grupo de baixo controle sobre o trabalho (Tabela 2). Na avaliação das questões que conferem o grau de controle ao trabalhador ${ }^{(6)}$, surpreendente foi o percentual de enfermeiros $(87 \%)$ que afirmou em men trabalho tenho pouca liberdade para decidir como eu devo fazế-lo (Tabela 1). O segundo fato evidenciou que $37,7 \%$ dos técnicos e auxiliares de enfermagem foram classificados com nível alto de controle no trabalho (Tabela 2).

Talvez a dinâmica do trabalho dos enfermeiros explique, em parte, o alto percentual para a pouca liberdade referida. Ou seja, existe todo um planejamento de atividades no transcorrer do turno de trabalho, porém, muitas vezes, eles não conseguem colocar em prática esse planejamento em razão de inúmeras interrupções (situações de emergência, falta de pessoal, entre outras), causando o sentimento de impotência e de pouco controle. Esse é um dos contextos singulares e variáveis do trabalho real $^{(8)}$. Em outras palavras, existe uma defasagem entre o prescrito e a realidade do trabalho da enfermagem, contribuindo para que os trabalhadores sejam afetados de diferentes formas pela situação laboral e reajam de diferentes modos.

No que se refere ao percentual de técnicos e auxiliares com alto controle, talvez o fato de muitos deles terem graduação em enfermagem possa explicar, também em parte, esse achado. Ou seja, sentem-se à vontade para tomar algumas decisões durante a execução das atividades. Nesse caso, as respostas estariam vinculadas à concepção do controle exercido na própria execução das atividades, desconsiderando ou não valorizando aspectos de definição mais estruturais do processo de trabalho (relações de poder dentro da instituição) ${ }^{(5)}$.

A organização do trabalho que neutraliza os comportamentos livres e criativos empobrece a atividade mental e coloca em perigo o corpo do trabalhador, podendo conduzi-lo a somatizações de ordem psíquica 
ou física ${ }^{(4)}$. Nesse sentido, corrobora a afirmação ${ }^{(6)}$ o baixo nível de controle sobre o planejamento e a execução das atividades laborais influencia nocivamente a saúde do trabalhador. Ou seja, ausência ou pouco controle pode ter efeitos na autoestima, ser fonte de estresse, insatisfação e de tensão no ambiente de trabalho. As repercussões disso sobre a saúde do trabalhador podem estar presentes, mesmo quando o comprometimento mais palpável e representado pelas alterações orgânicas não seja ainda visível ${ }^{(5-6)}$.

A avaliação do controle no trabalho da enfermagem nos remete à reflexão sobre o processo de formação dos profissionais dessa área ao longo da história. Tal processo produziu divisões, fragmentações e subordinações dentro da própria profissão: as ladies nurses e as nurses $^{(9)}$. As primeiras, eram formadas para o desempenho das atividades de supervisão e de ensino; as segundas, eram treinadas para o cuidado direto ao paciente e subordinadas à supervisão e coordenação das primeiras, raramente interferindo nos processos de tomada de decisão.

A formação profissional por si só determina maior ou menor grau de controle ao profissional, à medida que configura a divisão de atribuições e tarefas entre os trabalhadores de enfermagem. Pode-se dizer que o processo de divisão do trabalho da enfermagem reproduziu as formas hegemônicas de estruturação mais gerais da vida no trabalho, fortemente voltadas para dividir, fragmentar, parcelar, hierarquizar e subordinar, tanto as tarefas e atividades, como as relações entre os indivíduos ${ }^{(5,10)}$.

Outro aspecto a ser destacado neste estudo foi o predomínio de baixa demanda psicológica entre técnicos e auxiliares de enfermagem. Era de se esperar o contrário, dado que, para essas categorias funcionais, a divisão do trabalho da enfermagem, geralmente, impõe um ritmo mais intenso de trabalho ${ }^{(11)}$.

$\mathrm{Na}$ enfermagem, o trabalho pode ocorrer, na maioria das vezes, pela distribuição de tarefas entre seus diferentes membros, segundo operações hierarquizadas. Levandose em conta a complexidade das atividades, são exigidas habilidades diferenciadas de cada trabalhador no manejo de instrumentos, métodos e processos ${ }^{(9)}$. Nesse grupo de trabalhadores, o enfermeiro assume para si a responsabilidade gerencial e passa a supervisionar o trabalho e a controlar o processo como um todo. A responsabilidade pela parte intelectual do trabalho fica, então, a cargo do enfermeiro, mas não o isenta de participar das ações que envolvam esforço físico. Já a parte manual, é executada pelos técnicos e auxiliares de enfermagem, cabendo-lhes o papel de executores de tarefas delegadas.

A enfermagem é o grupo da saúde que assiste o paciente durante as 24 horas do dia, é natural que entre em contato com as necessidades decorrentes da condição humana dessas pessoas (comer, dormir, higiene pessoal e ambiente confortável $)^{(10)}$. Assim, os trabalhadores de enfermagem envolvem-se nesse cotidiano e assumem como suas todas essas responsabilidades e passam, inclusive, a serem cobrados por isso, tanto pelos pacientes como pelos médicos, familiares e pela administração dos serviços.

Então, na execução dessas atividades, sobretudo os técnicos e auxiliares necessitam desenvolver rotineiramente ações que envolvam um alto custo energético, como por exemplo; levantar, sustentar e transportar doentes e outras cargas; arrumar leitos; fazer curativos, por vezes, assumindo posições incômodas; fazer diversos deslocamentos; permanecer em uma postura quase sempre em pé; manter e transportar o peso do doente nas trocas de decúbito, na passagem maca-leito, leitocadeira, leito-banheiro. No serviço hospitalar, o trabalho da enfermagem caracteriza-se por ser intensivo que exige de seus membros alta produtividade em tempo limitado. A forma de organização e a quantidade de tempo destinadas ao trabalho assistencial hospitalar podem causar violência psicológica e ter efeito devastador e de longa duração na saúde dos trabalhadores ${ }^{(2)}$.

Neste estudo, a análise das interações entre os níveis de demandas psicológicas e de controle sobre o trabalho revelou que os trabalhadores de enfermagem encontravam-se predominantemente na categoria de trabalho passivo (Tabela 5). O MDC aponta que o trabalho passivo é a segunda exposição mais problemática para a saúde. A ausência de desafios significantes pode conduzir ao declínio na atividade global do indivíduo e a redução da capacidade de produzir soluções para as atividades e os problemas enfrentados ${ }^{(6)}$.

A divisão de tarefas entre técnicos e auxiliares de enfermagem (os que medicam; os que higienizam; os que transportam...), traz monotonia e repetitividade, podendo provocar desgaste físico e mental, bem como nutrir sentimentos de apatia nesses trabalhadores.

Ao comparar este trabalho com três estudos nacionais ${ }^{(5,12,13)}$ que utilizaram o MDC em investigações com trabalhadores de enfermagem em ambiente hospitalar, observou-se que: o primeiro estudo ${ }^{(5)}$ realizado um hospital da Bahia, os percentuais foram similares para trabalho passivo $(28,4 \%)$ e baixa exigência $(24,5 \%)$, ao passo que os demais apresentaram proporções inversas (trabalho ativo 19,3\% e alta exigência 27,8\%); o segundo ${ }^{(12)}$ com trabalhadores de enfermagem de unidades críticas de um hospital de Lajeado-RS verificou percentual mais elevado para trabalho em alta exigência $(44,1 \%)$ e menores percentuais para trabalho ativo (22\%), trabalho passivo $(19,7 \%)$ e trabalho em baixa exigência $(14,2 \%)$ e, o terceiro $^{(13)}$ com trabalhadores de enfermagem de um hospital municipal do Rio de Janeiro, revelou menor 
freqüência de trabalhadores nos grupos trabalho passivo $(16,6 \%)$ e alta exigência $(17,9 \%)$ e proporções mais elevadas nos grupos trabalho ativo $(39,1 \%)$ e baixa exigência $(26,6 \%)$.

Tais estudos evidenciam percentuais importantes de trabalhadores em trabalho de alta exigência, sobretudo para trabalhadores de enfermagem atuantes em unidades críticas. Fato que vem confirmar o trabalho da enfermagem, como um dos trabalhos da área da saúde com alto risco para agravo à saúde ${ }^{(14)}$. Pois, como estabelece o MDC - trabalho realizado em condições de alta exigência é nocivo à saúde dos trabalhadores, sendo um preditor da maioria das reações adversas produzidas no ambiente laboral ${ }^{(6)}$.

No entanto, vale ressaltar as limitações inerentes à utilização de uma escala que não foi criada com o objetivo de mensurar experiências estressantes do trabalho em um grupo específico de trabalhadores, como é o caso dos trabalhadores de enfermagem. Nesse sentido, alguns autores $^{(15)}$ questionam a aplicabilidade do MDC em certas áreas, especialmente, aquelas que requerem interação com pessoas, como é o caso de trabalhadores da área da saúde. Para esses autores ${ }^{(15)}$, a principal limitação do MDC diz respeito ao fato de que ele não necessariamente leva em conta as peculiaridades da natureza do trabalho que envolve relacionamento entre pessoas, uma vez que foi originalmente desenvolvido para utilização em indústrias, embora tenha a intenção de um uso generalizado.

Assim, algumas perguntas utilizadas na escala deveriam ser avaliadas com cuidado no contexto do processo de trabalho da enfermagem, como é o caso, por exemplo, do item o tempo para a realização das minhas atividades é suficiente. Como o trabalho da enfermagem tem continuidade nas 24 horas, a questão sobre o tempo para realização das tarefas parece não atingir o objetivo de medir a demanda psicológica. Quando um trabalhador não consegue terminar tal atividade, o colega do plantão seguinte a conclui. Com isso, a falta de tempo acaba por não representar, o que o modelo pretende medir (efeito danoso).

Também, no que se refere ao controle, o item men trabalho envolve muita repetitividade pode não estar necessariamente relacionado ao uso de habilidades no processo de trabalho, tal como postula o MDC. Alguns aspectos do ambiente hospitalar poderiam atenuar os efeitos maléficos dessa repetição. Dentre eles, poderia se destacar o fato de que essa repetitividade, em geral, envolve o cuidado de enfermagem. Em outras palavras, mesmo que o profissional de enfermagem execute somente um tipo de tarefa, como por exemplo, curativos durante todo o turno de trabalho, entre a repetição de um movimento e outro com a pinça, existe o interagir com o paciente ou familiar. Desta forma, também essa questão pode ficar prejudicada.

\section{CONCLUSÕES}

Os indicadores de demandas psicológicas e de controle sobre o trabalho entre trabalhadores de enfermagem, segundo o MDC, evidenciaram maior percentual de trabalhadores de enfermagem no grupo de trabalho passivo. Estão expostos a maior grau de desgaste - trabalho em alta exigência, 21,2\% dos trabalhadores, sobretudo técnicos e auxiliares de enfermagem.

O MDC foi um importante instrumento teóricometodológico na investigação dos aspectos psicossociais do trabalho da enfermagem. Por meio dele, observa-se que são diversos os elementos no ambiente laboral que podem representar potenciais estímulos de estresse e tensão ao profissional de enfermagem. Por exemplo: sobrecarga de trabalho, fragmentação das tarefas, pressão no tempo, condições laborais inadequadas, relacionamentos conflituosos, repetitividade e monotonia, intensividade e ritmo excessivo de trabalho que se mantêm por longos períodos, contribuem para a ocorrência de doenças ocupacionais. Novos estudos utilizando o MDC podem contribuir para a melhoria das condições de qualidade de vida e saúde dos trabalhadores de enfermagem. Da mesma forma, é necessário destacar que, após ter-se um pouco mais de conhecimento sobre essa metodologia, foi percebido que alguns aspectos merecem uma investigação ampliada, pois outras variáveis não avaliadas poderiam enriquecer os dados encontrados.

Enfim, medidas que visem a reduzir as demandas psicológicas provenientes do trabalho e que permitam maior flexibilidade e autonomia ao trabalhador de enfermagem são necessárias. Esses aspectos competem, tanto aos gestores, como aos próprios trabalhadores de enfermagem e aos pesquisadores. Algumas dessas medidas poderiam ser, respectivamente: gestão participativa, no sentido dos trabalhadores de enfermagem atuarem de forma integrada para apontarem caminhos na resolução dos problemas relativos à organização e ao processo de trabalho da enfermagem; construção coletiva de ações de promoção à saúde e de prevenção em um processo contínuo de (re)conhecimento das situações de risco presentes no ambiente laboral, e desenvolvimento de pesquisas que objetivem verificar associações entre as exigências do trabalho e a ocorrência de desordens físicas e psicológicas entre trabalhadores de enfermagem. Essas medidas poderão contribuir de forma efetiva para a melhoria das condições de saúde e trabalho da enfermagem. 


\section{REFERÊNCIAS}

1. Magnano TSBS, Lisboa MTL, Griep RH. Trabalho da enfermagem e distúrbio musculoesquelético: revisão das pesquisas sobre o tema. Esc Anna Nery Rev Enferm. 2008;12(3):560-5.

2. Costa ALRC, Marziale MHP. Relação tempo-violência no trabalho de enfermagem em Emergência e Urgência. Rev Bras Enferm. 2006; 59(3):337-43.

3. Vieira LC, Guimarães L, Martins D. O estresse ocupacional em enfermeiros. In: Guimarães LAM, Grubtis S, organizadores. Série saúde mental e trabalho. São Paulo: Casa do Psicólogo; 1999. Vol 1. p. 169-85.

4. Dejours C. A carga psíquica do trabalho. In: Dejours C, Abdoucheli E, Jayet C. Psicodinâmica do trabalho: contribuições da escola dejouriana à análise da relação prazer, sofrimento e trabalho. São Paulo: Atlas; 1994. p. 21-32.

5. Araújo TM. Trabalho e distúrbios psíquicos em mulheres trabalhadoras de enfermagem [tese]. Salvador: Instituto de Saúde Coletiva da Universidade Federal da Bahia; 1999.

6. Karasek R, Theörell T. Healthy work: stress, productivity, and the reconstruction of working life. New York: Basic Books; c1990.

7. Araújo TM, Karasek R. Validity and reliability of the job content questionnaire in formal and informal jobs in Brazil. Scand J Work Environ Health Suppl. 2008;34(6):52-9.

8. Brito JC. Trabalho prescrito e trabalho real. In: Escola Politécnica de Saúde Joaquim Venâncio, Estação de Trabalho Observatório de Técnicos em Saúde, organizadores. Dicionário da educação profissional em saúde. Rio de Janeiro:
EPSJV; 2006. p. 282-94.

9. Nightingale F. Notas sobre enfermagem: o que é e o que não é. São Paulo: Cortez; 1989.

10. Pires D. Processo de trabalho em saúde, no Brasil, no contexto das transformações atuais na esfera do trabalho: estudo em instituições escolhidas [tese]. Campinas: Instituto de Filosofia e Ciências Humanas da Universidade Estadual de Campinas; 1996.

11. Gonzales RMB. Sofrimento na práxis da enfermagem: real ou deslocado em seu sentido? [tese]. Florianópolis: Centro de Ciências da Saúde da Universidade Federal de Santa Catarina; 2000.

12. Amaral TR. Dimensões psicossociais do trabalho da enfermagem e os distúrbios psíquicos menores em unidades críticas [dissertação]. Florianópolis: Curso de Pós-graduação em Enfermagem da Universidade Federal de Santa Catarina; 2006.

13. Silva JLL. Estresse e transtornos mentais comuns em trabalhadores de enfermagem [dissertação]. Rio de Janeiro: Programa de Pós-Graduação em Enfermagem da Universidade Federal do Estado do Rio de Janeiro; 2007.

14. Jodas DA, Hadad MCL. Síndrome de Burnout em trabalhadores de enfermagem de um pronto socorro de hospital universitário. Acta Paul Enferm. 2009; 22(2):192-7.

15. Söderfeldt B, Söderfeldt M, Muntaner C, O'Campo P, Warg LE, Ohlson CG. Psychosocial work environment in human service organizations: a conceptual analysis and development of the demand-control model. Soc Sci Med. 1996;42(9):1217-26. 